\title{
Phase transitions and gluodynamics in 2-colour matter at high density
}

\author{
Tamer Boz ${ }^{1}$, Seamus Cotter ${ }^{1}$, Leonard Fister ${ }^{1}$, Dhagash Mehta ${ }^{2}$, and Jon-Ivar Skullerud ${ }^{1}$ \\ 1 Department of Mathematical Physics, National University of Ireland Maynooth, Maynooth, County Kildare, Ireland \\ 2 Department of Physics, Syracuse University, Syracuse, NY 13244, USA
}

Received: date / Revised version: date

\begin{abstract}
We investigate 2-colour QCD with 2 flavours of Wilson fermion at nonzero temperature $T$ and quark chemical potential $\mu$, with a pion mass of $700 \mathrm{MeV}\left(m_{\pi} / m_{\rho}=0.8\right)$. From temperature scans at fixed $\mu$ we find that the critical temperature for the superfluid to normal transition depends only very weakly on $\mu$ above the onset chemical potential, while the deconfinement crossover temperature is clearly decreasing with $\mu$. We find indications of a region of superfluid but deconfined matter at high $\mu$ and intermediate $T$. The static quark potential determined from the Wilson loop is found to exhibit a 'string tension' that increases at large $\mu$ in the 'deconfined' region. The electric (longitudinal) gluon propagator in Landau gauge becomes strongly screened with increasing temperature and chemical potential. The magnetic (transverse) gluon shows little sensitivity to temperature, and exhibits a mild enhancement at intermediate $\mu$ before becoming suppressed at large $\mu$.
\end{abstract}

PACS. $11.15 \mathrm{Ha}$ Lattice gauge theory $-12.38 \mathrm{Aw}$ Lattice QCD calculations - 21.65Qr Quark matter $12.38 \mathrm{Mh}$ Quark-gluon plasma

\section{Introduction}

The properties and phase diagram of quantum chromodynamics (QCD) at large baryon density remain largely unknown, despite substantial theoretical efforts. One important reason for this is the failure of traditional Monte Carlo methods for lattice QCD at nonzero density, due to the infamous sign problem. While progress has been made in the region of high temperature $T$ and moderate baryon chemical potential $\mu_{B}$, the region of low $T$ and large $\mu_{B}$ remains inaccessible to Monte Carlo simulations. 1

There are, however, QCD-like theories which are not affected by the sign problem, at least for an even number of flavours $N_{f}$, among them QCD with gauge groups $\mathrm{SU}(2)$ $\left(\mathrm{QC}_{2} \mathrm{D}\right)$ [5, 6, 7, or $\mathrm{G}_{2}$ [8, with nonzero isospin density [9] or with adjoint fermions 10,11. These theories allow first-principles lattice simulations and may hence be used as benchmarks for other methods that are not encumbered by the sign problem, but which may involve uncontrolled approximations. Such methods may include model studies such as NJL and quark-meson models (possibly augmented by a Polyakov loop potential); effective theories valid for example for heavy quarks or at high density; or functional methods such as the functional renormalisation group (FRG), Dyson-Schwinger equations (DSEs)

\footnotetext{
${ }^{1}$ Some progress has recently been made using complex Langevin [1,2] and other methods [3,4, but neither have as yet been shown to work for QCD.
}

or n-particle irreducible (nPI) methods, which rely on assumptions about higher-order vertices.

Among these QCD-like theories, $\mathrm{QC}_{2} \mathrm{D}$ is the simplest, both mathematically and computationally; however, it shares important properties with full QCD, in particular a hadronic phase and deconfinement. Therefore, it has been adressed in various approaches like analytic continuation methods at high temperature and low density [12,13. 14, chiral effective theories $15,16,17,18,19,20,21,22,23$, 24, 25, 26, 27, 28, (Polyakov-)Nambu-Jona-Lasinio or (Polyakov)Quark-Meson(-Diquark) models [29,30, 31, 32, 33, 34, 35, 36, 37,38, 39, Dyson-Schwinger equations [40] and lattice gauge theory [41,42, 43, 44, 45, 46, 47,5, 6, 48. From these studies a conjectured phase diagram in the $(\mu, T)$ plane has emerged. At small values for chemical potential and temperature, quarks are confined but their chiral symmetry is broken which leads to a non-vanishing chiral condensate as in full QCD, hence a hadronic phase. However, the Pauli-Gürsey symmetry of $\mathrm{QC}_{2} \mathrm{D}$ leads to a characteristic difference. In the chiral limit for two flavours, the spontaneous symmetry breaking by the chiral condensate necessitates 5 (massless) Nambu-Goldstone bosons, three of which are the pions, while the remaining two are the diquark and antidiquark. This pattern is realised approximately also for small quark masses. The (anti-)diquarks play the role of the baryons, hence having Bose-Einstein statistics in $\mathrm{QC}_{2} \mathrm{D}$. For vanishing temperature but increasing chemical potential the Silver Blaze property 49 dictates that 
observables must not change up to the threshold of the quark chemical potential $\mu=m_{\text {baryon }} / N_{c}$, hence half of the pion mass for two colour matter. Above this threshold, the different statistics of the baryons lead to a qualitatively different phase structure compared to full QCD: For $\mu>m_{\pi} / 2$ the baryons condense and form a BEC of diquarks $\langle q q\rangle \neq 0$, which possibly turns into a BCS condensate at high densities, in contrast to the coloursuperconductor picture in full QCD. For increasing temperatures also $\mathrm{QC}_{2} \mathrm{D}$ is expected to exhibit a crossover transition to a deconfined phase, like full QCD. This transition is signalled by the rise of the Polyakov-loop $\langle L\rangle$. The structure of at least three distinct phases is characterised by vanishing or nonvanishing diquark condensate $\langle q q\rangle$ and Polyakov loop $\langle L\rangle$ and can be summarised by

- a vacuum or hadronic phase at low $T$ and $\mu$, characterised by vanishing quark number density $n_{q}$ and with $\langle q q\rangle=0,\langle L\rangle \approx 0$

- a superfluid, confined (quarkyonic) region at low $T$ and intermediate to large $\mu$, with $\langle L\rangle \approx 0,\langle q q\rangle \neq 0$;

- a deconfined quark-gluon plasma at high $T$, with $\langle L\rangle \neq$ $0,\langle q q\rangle=0 ;$ and possibly

- a deconfined, superfluid region at large $\mu$ and intermediate $T$, with $\langle L\rangle \neq 0,\langle q q\rangle \neq 0$.

A quarkyonic phase was first conjectured in [50, in the context of large $N_{c}$ and defined as confined and chirally symmetric. It was later found 51 that chiral symmetry may be broken in unconventional ways, and it seems more appropriate instead to define quarkyonic as a state of matter where (weakly interacting) quarks form the bulk degrees of freedom, but which remains confined, ie all excitations are hadronic. Quarkyonic matter in $\mathrm{QC}_{2} \mathrm{D}$ was studied in [34, and in 6] evidence for a quarkyonic region was presented from lattice simulations.

In the present paper we extend the analysis of [7, where rough estimates for the phase boundaries between these regions of $\mathrm{QC}_{2} \mathrm{D}$ were presented. We study the phase transitions in more detail and attempt to pinpoint their location. Since we are using Wilson fermions and quite heavy quarks, we are not in a position to study chiral symmetry directly. An exploratory attempt to adress this issue was made in [7].

We will also attempt to cast further light on the confining properties of the theory at low temperature and the nature of the putative deconfinement transition at high density, by computing the static quark potential in the low-temperature region.

In contrast to quantities which may not be directly comparable between theories, the effects of the medium on low order Green functions in $\mathrm{QC}_{2} \mathrm{D}$ may provide a reliable guideline to full QCD. Quark and gluon correlation functions are of great interest, as the theory can be fully expressed in terms of these. Propagators play a predominant role, in particular in continuum descriptions, and in some cases their behaviour suffices to shed light on the critical physics of the phase diagram, e.g. the deconfinement transition [52,53,54,55. In this paper, we will study how the gluon propagator responds to both temperature and quark chemical potential.
In Sec. 2 we set out the details of our lattice simulations, including the action, parameters and lattice volumes used. Then, in Sec. 3 we study the superfluid to normal and deconfinement transition by performing a temperature scan at 3 different values of the chemical potential. The response of the static quark potential to $\mu$ is investigated in Sec. 4, while in Sec. 5 results for the gluon propagator are reported. Appendix $\mathrm{A}$ contains some further details about the diquark source extrapolation of the superfluid condensate. Preliminary results for the gluon propagator have been reported in [56,57, and for the static quark potential in 58.

\section{Simulation details}

We use a standard Wilson gauge action with two flavours of unimproved Wilson fermion, with the addition of a diquark source term to lift the low-lying eigenmodes and allow a controlled study of diquark condensation effects. Further details about the action and the simulation method can be found in [5,6,7]. The results obtained will depend on the diquark source $j$; in the end the $j \rightarrow 0$ limit must be taken to obtain 'physical' results 2

We use the same parameters as in 6, 7], namely $\beta=$ $1.9, \kappa=0.168$, corresponding to a lattice spacing $a=$ $0.178(6) \mathrm{fm}$ and a pion mass $a m_{\pi}=0.645(8)$, or $m_{\pi}=$ $717(25) \mathrm{MeV}$. The lightest baryon, the scalar diquark, is degenerate with the pion in the vacuum, and at zero temperature we therefore expect an onset transition to a superfluid phase at $m=m_{\pi} / 2$. This has been corroborated in previous simulations [5, 6, 7 .

In addition to the ensembles used and described in [7], we have generated gauge configurations on $16^{3} \times N_{\tau}$ lattices with $N_{\tau}=4-20$, in order to study in detail the thermal transitions at $a \mu=0.35,0.4,0.5$ and 0.6. The details of these ensembles are given in table 1. For most temperatures, two diquark sources $j a=0.02$ and 0.04 have been used, enabling us to perform a linear extrapolation to the $j=0$ limit. In the region of the superfluid to normal transition, where a linear extrapolation is known to be invalid, two additional $j$-values have been added to allow for a controlled extrapolation.

\section{Phase transitions}

\subsection{Superfluid to normal transition}

Figure 1 shows the order parameter for superfluidity, the (unrenormalised) diquark condensate

$$
\langle q q\rangle=\left\langle\psi^{2 t r} C \gamma_{5} \tau_{2} \psi^{1}-\bar{\psi}^{1} C \gamma_{5} \tau_{2} \bar{\psi}^{2 t r}\right\rangle,
$$

\footnotetext{
${ }^{2}$ In cases where model studies could be carried out with $j \neq 0$ one might also compare directly results for nonzero $j$; however, most other studies will not contain any explicit diquark source term, so the $j \rightarrow 0$ limit is crucial.
} 


\begin{tabular}{|c|c|c|c|c|c|c|c|c|c|c|c|c|c|c|c|c|}
\hline \multirow[b]{2}{*}{$\mu a$} & \multirow[b]{2}{*}{ ja } & \multicolumn{15}{|c|}{$N_{\tau}$} \\
\hline & & 20 & 18 & 16 & 15 & 14 & $\overline{13}$ & 12 & 11 & 10 & 9 & 8 & 7 & 6 & 5 & $\overline{4}$ \\
\hline 0.35 & 0.02 & & & 270 & & & 370 & 250 & 270 & 510 & 560 & 800 & 520 & 1100 & 300 & 250 \\
\hline 0.35 & 0.04 & & & 250 & & 270 & & 4550 & 710 & 500 & 500 & 675 & 510 & 900 & 300 & 250 \\
\hline 0.4 & 0.02 & & & 270 & & & 250 & 500 & 500 & 500 & 550 & 1000 & 250 & 250 & 300 & \\
\hline 0.4 & 0.03 & & & & & & & & 250 & 280 & 300 & & & & & \\
\hline 0.4 & 0.04 & & & & & & 1020 & 1080 & 1050 & 1000 & 1000 & 1050 & 1000 & 1000 & 1000 & 1000 \\
\hline 0.4 & 0.05 & & & & & & & & 250 & 240 & 216 & & & & & \\
\hline 0.5 & 0.02 & & & 280 & & & & 512 & 250 & 255 & 275 & 1000 & 250 & 300 & & \\
\hline 0.5 & 0.03 & & & & & & & & 280 & 270 & 280 & 1000 & & & & \\
\hline 0.5 & 0.04 & 3075 & 3020 & 2570 & 3835 & 3340 & 3200 & 3240 & 2620 & 1000 & 1000 & 1050 & 1200 & 1000 & 1200 & 1000 \\
\hline 0.5 & 0.05 & & & & & & & & 300 & 270 & 270 & & & & & \\
\hline 0.6 & 0.02 & & & 350 & & 320 & & 310 & 300 & 300 & 300 & 1000 & 300 & 300 & & \\
\hline 0.6 & 0.03 & & & & & & & & 290 & 280 & 280 & & & & & \\
\hline 0.6 & 0.04 & 4200 & & 2300 & 2210 & 1840 & 1000 & 1128 & 1005 & 1000 & 1040 & 1200 & 1020 & 1000 & & \\
\hline 0.6 & 0.05 & & & & & & & & 255 & 300 & 300 & & & & & \\
\hline
\end{tabular}

Table 1. Number of trajectories for different temperatures, $T=1 /\left(a N_{\tau}\right)$, chemical potentials $\mu$ and diquark sources $j$. All trajectories have average length 0.5 , and the spatial lattice size is $N_{s}=16$ in all cases.

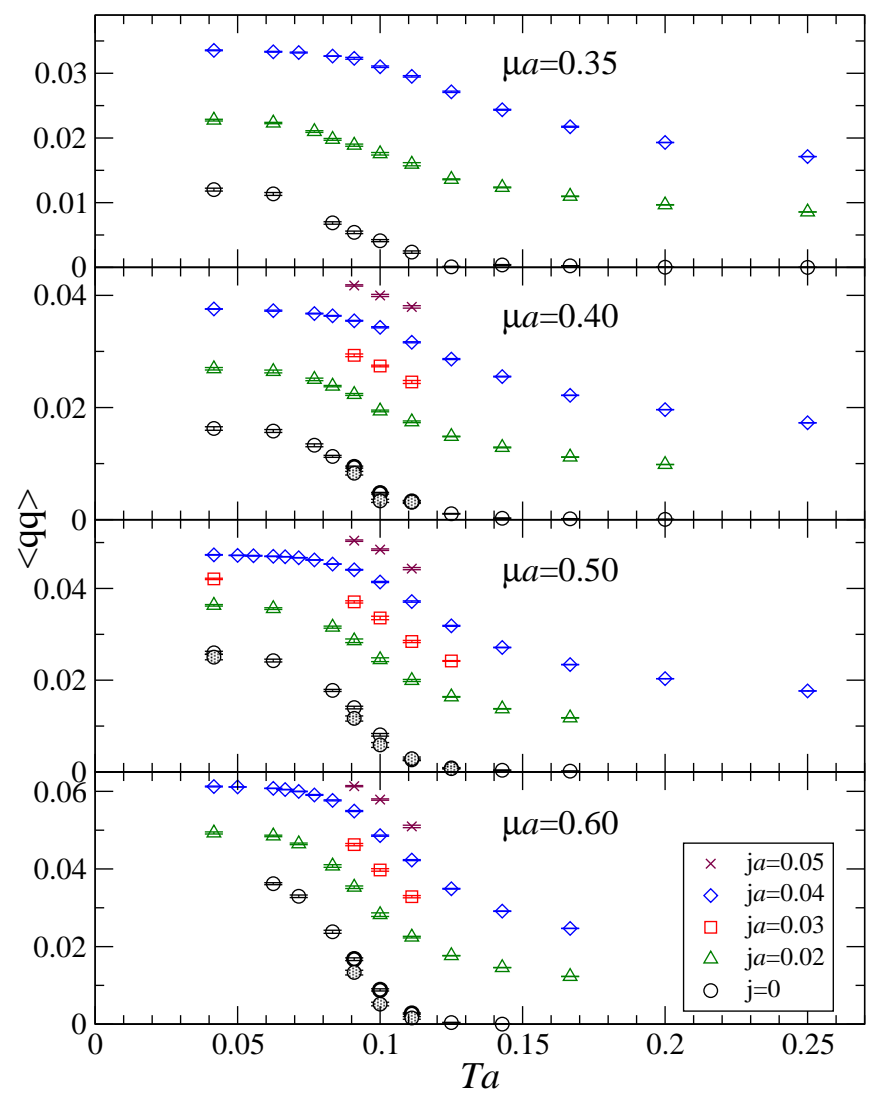

Fig. 1. Diquark condensate $\langle q q\rangle$ as a function of temperature $T$ for chemical potential $\mu a=0.35,0.4,0.5,0.6$ (top to bottom). The circles are data extrapolated to $j=0$ using a linear Ansatz for $j a \leq 0.04$; the shaded circles denote the results of a linear extrapolation using $j=0.02,0.03$ only.

as a function of the temperature $T$, for $\mu a=0.35,0.4,0.5$ and 0.6. Also shown are the results of a linear extrapolation to $j=0$. Note that renormalisation will only amount to multiplying these data by a $\mu$ - and $T$-independent con- stant, and will hence not change the $\mu$ - and $T$-dependence of the results.

We can clearly observe a transition from a superfluid phase, characterised by $\langle q q\rangle \neq 0$, at low temperature, to a normal phase with $\langle q q\rangle=0$ at high temperature, with a transition in the region $0.08 \lesssim T a \lesssim 0.12$ for all three values of $\mu$. In an attempt to more precisely locate the transition, we have performed simulations with 4 different $j$-values in the transition region, which may allow for a controlled $j \rightarrow 0$ extrapolation. However, none of the functional forms we have used give satisfactory results. Details of these extrapolations are given in Appendix A.

With our current data at only a single volume we are not in a position to determine the order of the transition, although it would be expected to be a second order phase transition in the universality class of the 3-dimensional XY model. This could be tested by attempting a universal fit of the data at $j \neq 0$ in the transition region to a scaling form given by the appropriate critical exponents: note that at fixed $\mu$, the diquark source $j$ would be the magnetic field variable in this scaling function. This would also provide an alternative method for determining $T_{s}$.

Because of the uncertainties in the $j \rightarrow 0$ extrapolation in the critical region, we have estimated the critical temperatures $T_{s}$ for the superfluid to normal transition by determining the inflection points for $\langle q q\rangle$ at $j a=0.02$ and 0.04 , and extrapolated the resulting values to $j=0$ using a linear Ansatz. The results are given in table 2. We see that $T_{s}$ is remarkably constant over the whole range of $\mu$ values considered. The indications are that the transition happens at a somewhat lower temperature at $\mu a=0.35$, but this point is already very close to the onset from vacuum to superfluid at $T=0, \mu_{o} a=m_{\pi} a / 2=0.32$, suggesting that $T_{s}(\mu)$ rises very rapidly from zero at $\mu=\mu_{o}$ before suddenly flattening off. 


\begin{tabular}{c|rrrr}
$a \mu$ & 0.35 & 0.40 & 0.50 & 0.60 \\
\hline$a T_{s}(0.04)$ & $0.121(6)$ & $0.108(2)$ & $0.111(5)$ & $0.102(6)$ \\
$a T_{s}(0.02)$ & $0.097(16)$ & $0.096(5)$ & $0.097(2)$ & $0.093(5)$ \\
\hline$a T_{s}$ & $0.073(24)$ & $0.084(8)$ & $0.083(5)$ & $0.083(6)$ \\
$T_{s}(\mathrm{MeV})$ & $82(27)$ & $94(9)$ & $93(6)$ & $93(7)$
\end{tabular}

Table 2. Inflection points $T_{s}(j)$ for $\langle q q\rangle(T)$ at $j a=0.04,0.02$ and critical temperature $T_{s}$ obtained from extrapolating $T_{s}(j)$ to $j=0$. The uncertainties are estimates of the systematic uncertainty in determining the inflection points and in the $j \rightarrow$ 0 extrapolation.

\subsection{Deconfinement transition}

The Polyakov loop $\langle L\rangle$ serves as the traditional order parameter for deconfinement in gauge theories, with $\langle L\rangle \neq 0$ signalling the transition to a deconfined phase. Strictly speaking, $\langle L\rangle$ is never zero in a theory with dynamical fermions, but it typically increases with temperature from a very small value in a fairly narrow region, which may be identified with the deconfinement transition region.

We will here take the pragmatic view in which $\mathrm{QC}_{2} \mathrm{D}$, like QCD, is considered to be confining at low $T$ and $\mu$. This is also reflected in the behaviour of the static quark potential, which will be studied in the following section: it rises linearly at intermediate distances, before string breaking sets in.

Unlike the diquark condensate, the renormalisation of the Polyakov loop does depend on temperature; specifically, the relation between the bare Polyakov loop $L_{0}$ and the renormalised Polyakov loop $L_{R}$ is given by

$$
L_{R}(T, \mu)=Z_{L}^{N_{\tau}} L_{0}\left(\frac{1}{a N_{\tau}}, \mu\right) .
$$

In order to investigate the sensitivity of our results to the renormalisation scheme, we have used two different conditions to determine the constant $Z_{L}$,

$$
\begin{array}{ll}
\text { Scheme A } & L_{R}\left(T=\frac{1}{4 a}, \mu=0\right)=1, \\
\text { Scheme B } & L_{R}\left(T=\frac{1}{4 a}, \mu=0\right)=0.5 .
\end{array}
$$

Scheme A is the scheme that was already used in [7]. Figure 2 shows $\langle L\rangle$ evaluated in both schemes, as a function of temperature. The Scheme B data have been multiplied by 2 to ease the comparison with the Scheme A data. Also shown are cubic spline interpolations of the data and the derivative of these interpolations, with solid lines corresponding to Scheme A and dotted lines to Scheme B.

At all $\mu$, we see a transition from a low-temperature confined region to a high-temperature deconfined region. In contrast to the diquark condensate, we see a clear, systematic shift in the transition region towards lower temperatures as the chemical potential increases. For all four $\mu$-values, the Polyakov loop shows a nearly linear rise as a function of temperature in a broad region, suggesting that the transition is a smooth crossover rather than a true phase transition. This is reinforced by the difference

\begin{tabular}{l|cc}
$\mu a$ & $T_{d} a$ & $T_{d}(\mathrm{MeV})$ \\
\hline 0.0 & $0.193(20)$ & $217(23)$ \\
0.35 & $0.140-0.220$ & $157-247$ \\
0.40 & $0.108-0.200$ & $121-225$ \\
0.50 & $0.080-0.200$ & $90-225$ \\
0.60 & $0.060-0.135$ & $67-152$
\end{tabular}

Table 3. Estimates for the deconfinement crossover temperature $T_{d}$ from the Polyakov loop at $j a=0.04$. The $\mu=0$ result is taken from [7.

between Scheme A and Scheme B, with the crossover occuring at higher temperatures in Scheme B. At $\mu=0$, the difference between the two schemes is small, but increases with increasing $\mu$, suggesting a broadening of the crossover.

Because of the smaller value of $Z_{L}$, our results for Scheme B are considerably less noisy than those for Scheme A. For this reason, we choose to define the crossover region to be centred on the inflection point from Scheme $\mathrm{B}$, with a width chosen such that it also encompasses the onset of the linear region from Scheme A. Our summary of transition temperatures taken from the $j a=0.04$ data is given in table 3. From Fig. 2 we see that at low $T$, the value of $\langle L\rangle$ increases as $j$ is reduced, and at $\mu a=0.6$, the crossover region will most likely move to smaller $T$ in the $j \rightarrow 0$ limit. However, we do not have sufficient statistics for $j a=0.02$ at low $T$ to make any quantitative statement about this.

\section{Static quark potential}

The potential between two static quarks (or a quarkantiquark pair), and in particular its asymptotic behaviour at large separations, has traditionally been taken as the tell-tale indicator, or even definition, of confinement of quarks [59]. A linearly rising potential has been observed in numerous lattice simulations, and has also formed the basis of successful phenomenological descriptions of bound states of heavy quarks. In QCD with dynamical quarks, the string will break at a finite distance, but at intermediate distances a linear rise can still be observed.

At high temperature, the potential is expected to exhibit Debye screening, and this has been observed in numerous calculations of the quark-antiquark free energy using Polyakov loop correlators. However, it is not yet clear how this quantity relates to the (complex) potential that appears in effective theories of heavy quarkonia at high temperature 60,61,62,63. Very recently, the static quark potential has also been determined from Wilson loops at high temperature 64; this does not show any screening for $T \lesssim T_{c}$.

There has also been some recent progress in determining the potential between heavy (finite mass) quarks at zero 65] and non-zero 66] temperature. Some properties of bound states of heavy quarks in $\mathrm{QC}_{2} \mathrm{D}$ at nonzero temperature and density were reported in 67]; a potential model description should reproduce these results. Here we 


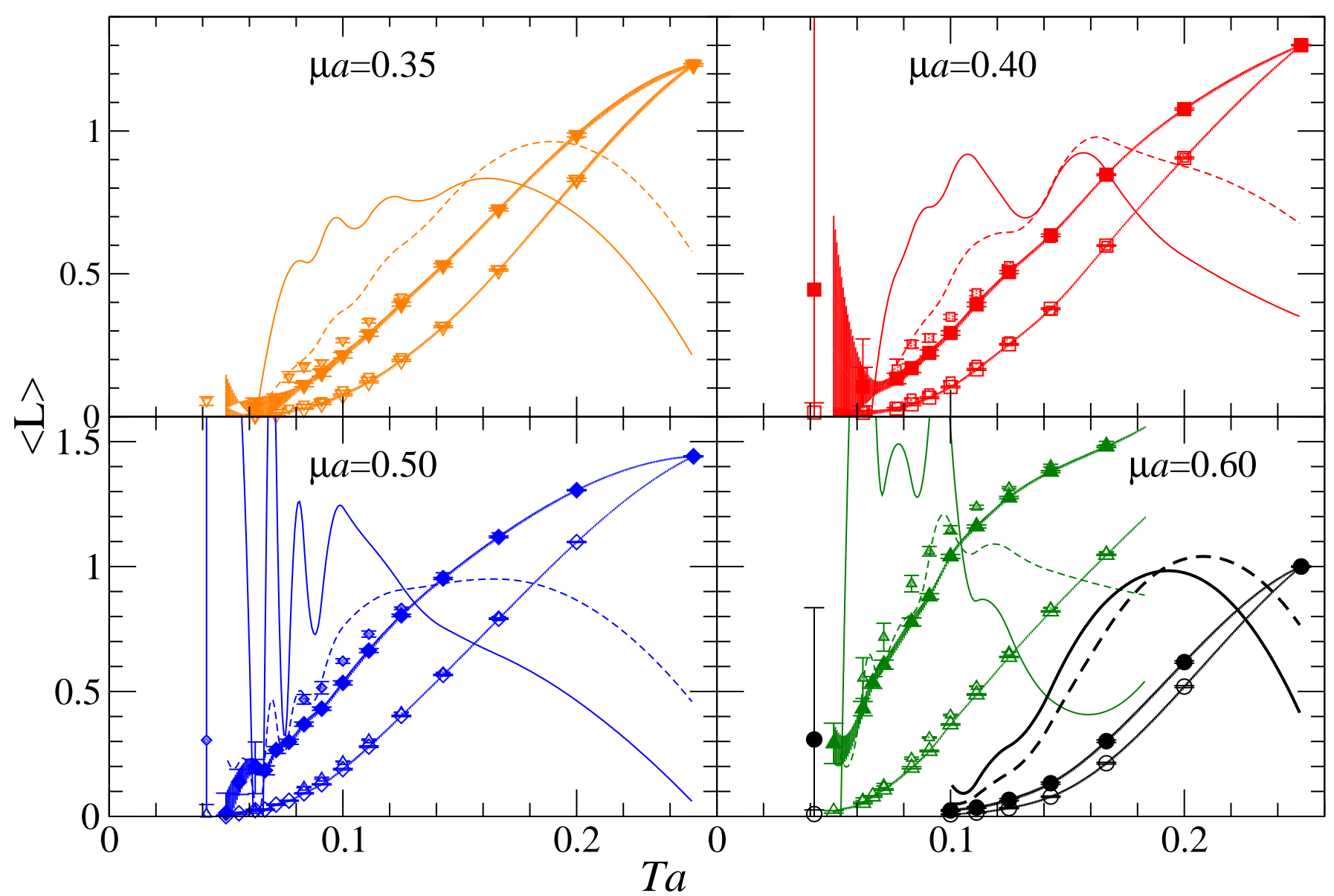

Fig. 2. The renormalised Polyakov loop $\langle L\rangle$ as a function of temperature $T$ for $j a=0.04$ and $\mu a=0.35,0.4,0.5,0.6$, with two different renormalisation schemes: Scheme A (solid symbols) and Scheme B (open symbols), see text for details. The solid (dashed) lines are the derivatives of cubic spline interpolations of the data points for Scheme A (B). The smaller, shaded symbols are results for $j a=0.02$. The black circles and thick lines in the bottom right panel are the $\mu=j=0$ results from 7 .

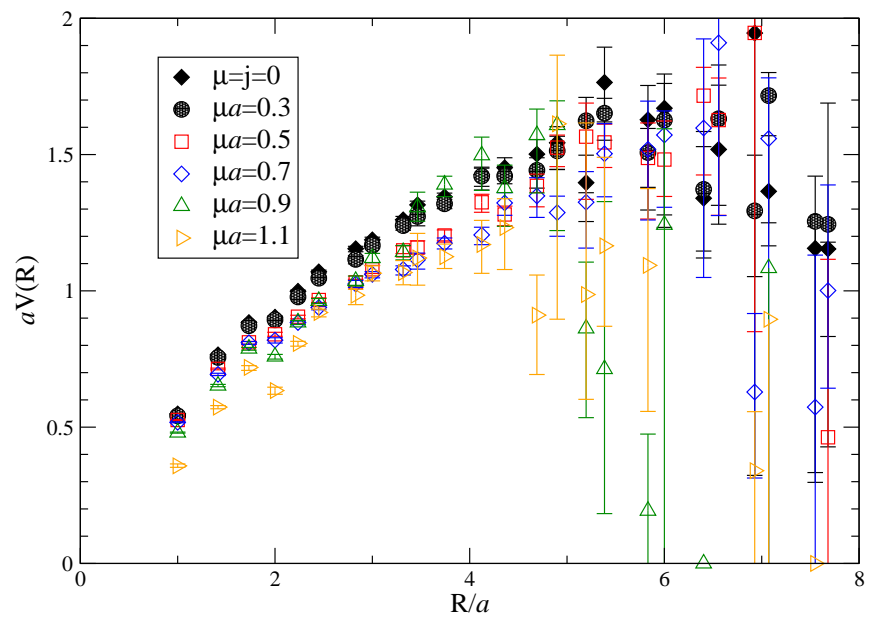

Fig. 3. The static quark potential computed from the Wilson loop, for the $12^{3} \times 24$ lattice and different values of $\mu$, with $j a=0.04$.

compute the static quark potential from Wilson loops for our lowest temperature, the $12^{3} \times 24$ lattices.
In fig. 3 we show the static quark potential computed from the Wilson loop at $N_{\tau}=24$, for $\mu a=0.3,0.5,0.7,0.9$ and 1.1. The data have been obtained from fits to the asymptotic form of the Wilson loop, $W(r, \tau)=C \exp (-V(r) \tau)$, assuming ground state dominance for $\tau \geq 3$. We find that as we enter the superfluid region, the potential becomes slightly flatter, but as $\mu$ is increased further no additional screening is observed, and at $\mu a=0.9$, which according to our analysis of the Polyakov loop should be in the deconfined region, the potential is consistent with the $\mu=0$ potential. This is in qualitative agreement with the pattern that was already observed in [5].

To quantify the variation of the static quark potential with $\mu$, we have performed a fit to the Cornell potential,

$$
V(r)=C(\mu, j)+\sigma(\mu, j) r+\frac{\alpha(\mu, j)}{r}
$$

and a screened potential with an exponential term to allow for screening effects and a decay of the linear part,

$$
V(r)=C(\mu, j)+\frac{\sigma(\mu, j) r}{B(\mu, j)} e^{-B r}+\frac{\alpha(\mu, j)}{r},
$$




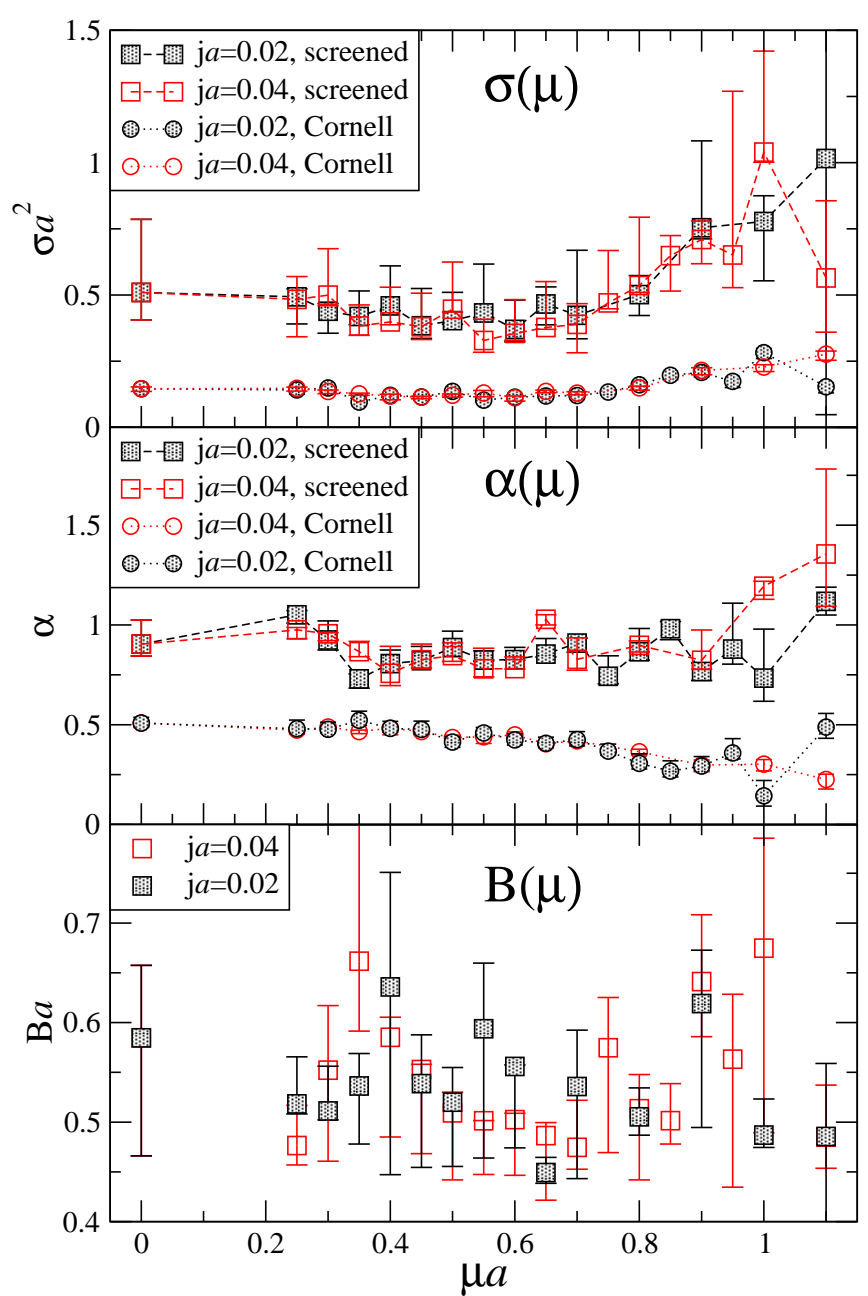

Fig. 4. The parameters $\sigma$ (top), $\alpha$ (middle) and $B$ (bottom) in the screened Cornell potential (4), as a function of the chemical potential $\mu$, for the $12^{3} \times 24$ lattice. Also shown are the results for $\sigma$ and $\alpha$ using the pure Cornell potential (3).

for each $\mu$ and $j$. The results for the "string tension" $\sigma$ and the Coulomb factor $\alpha$ are shown in fig. 4. For $\mu a<0.6$ we do not see any systematic trend, but for larger $\mu$ we find that $\sigma$ increases with $\mu$. We also see that there is no significant dependence on the diquark source $j$. The exponential term appears to be insensitive to $\mu$, and is clearly non-zero already at $\mu=0$, and should therefore not be interpreted as a screening mass. For the same reason, the values for $\sigma$ and $\alpha$ from the two fits cannot be directly compared.

In 68 it was observed that the static quark potential extracted from Wilson loops in QCD at $\mu=0$ is more weakly screened at intermediate temperature than the free energy determined from Polyakov loop correlators. Our results at large $\mu$ appear consistent with this. There are, however, several possible complicating factors:

1. The transition may be to a medium characterised by long-range interactions, rather than by colour screening. If that is the case, one would expect typical correlation lengths to also grow with $\mu$. Reconciling an unscreened potential with a nonzero Polyakov loop remains a challenge, though.

2. The quark-antiquark potential may be screened at large distances, but this is not observed in the Wilson loop because of poor overlap with the relevant states. This corresponds to the standard scenario at low temperature, where explicit mesonic states must be introduced to observe string breaking 69. Clearly, our observation of a linearly rising potential (area law for the Wilson loop) does not prove that the medium is confining. The increasing slope could however be indicative of a large internal energy for static quark-antiquark pairs at intermediate distances.

In 67. it was found that the binding energy of heavy quarkonia in $\mathrm{QC}_{2} \mathrm{D}$ increases up to $\mu a \approx 0.7$, and decreases again beyond that. This appears to run counter to the conjecture above, in which the quarkonium might be expected to become more strongly bound.

3. A more pessimistic scenario is that the whole region of $\mu a>0.6$ could be dominated by lattice artefacts. Unfortunately our previous data at $\beta=1.7$ [5] are probably outside the scaling region so a comparison with those is likely to be not very revealing. Simulations at smaller $a$, which are underway, should confirm or rule out this scenario.

Computing the static quark potential using Polyakov loop correlators rather than Wilson loops might yield further insight into this issue. However, the Polyakov loop correlator suffers from the same signal to noise problem at low $T$ (large $N_{\tau}$ ) as the Polyakov loop itself, and we have not been able to obtain any signal for $N_{\tau}=24$ except for $\mu a \gtrsim 0.9$. Results for higher temperatures using both the Wilson loop and Polyakov loop correlators will be presented in a future publication.

\section{Gluon propagator}

In this section we extend previous studies [5,56, 57] and present results for in-medium gluon propagators, where we study the dependence on both parameters, chemical potential and temperature. In Landau gauge only the transverse part of the vacuum propagator is non-zero. However, the external parameters break manifest Lorentz invariance, hence the gluon propagator $D$ must be decomposed into chromoelectric and chromomagnetic modes, $D_{E}$ and $D_{M}$, respectively,

$$
D_{\mu \nu}=P_{\mu \nu}^{M} D_{M}+P_{\mu \nu}^{E} D_{E},
$$

where the individual dependence on (discrete) temporal and spatial momenta has been omitted. The projectors on the longitudinal and transversal spatial subspaces, $P_{\mu \nu}^{E}$ and $P_{\mu \nu}^{M}$, are defined by

$$
\begin{aligned}
& P_{\mu \nu}^{M}\left(q_{0}, \boldsymbol{q}\right)=\left(1-\delta_{0 \mu}\right)\left(1-\delta_{0 \nu}\right)\left(\delta_{\mu \nu}-\frac{q_{\mu} q_{\nu}}{\boldsymbol{q}^{2}}\right), \\
& P_{\mu \nu}^{E}\left(q_{0}, \boldsymbol{q}\right)=\left(\delta_{\mu \nu}-\frac{q_{\mu} q_{\nu}}{q^{2}}\right)-P_{\mu \nu}^{M}\left(q_{0}, \boldsymbol{q}\right) .
\end{aligned}
$$



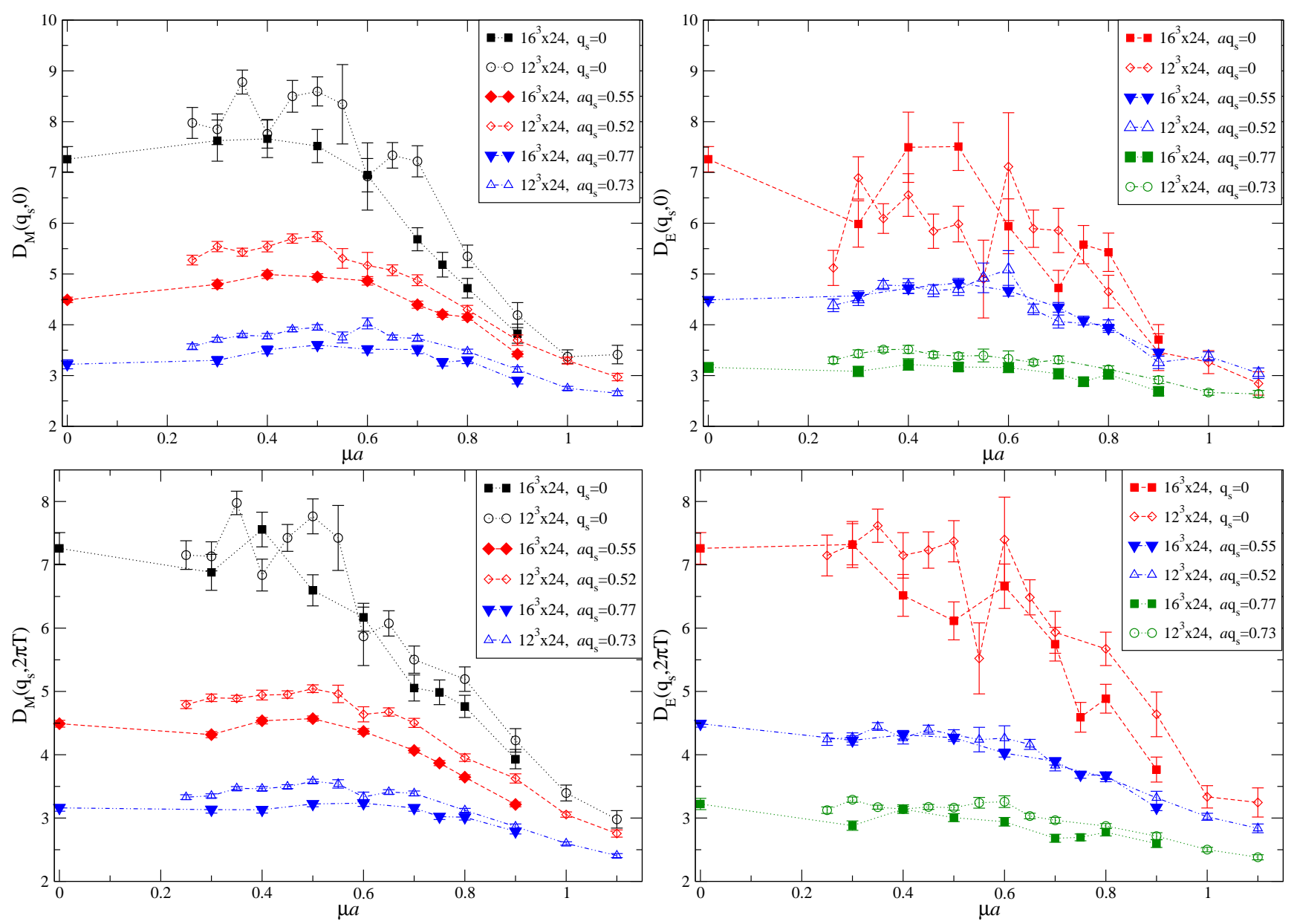

Fig. 6. The zeroth (top) and first (bottom) Matsubara mode of the magnetic (left) and electric (right) gluon propagator as a function of chemical potential $\mu$ for selected values of the spatial momentum $q_{s}$, for $N_{\tau}=24$, different spatial volumes.

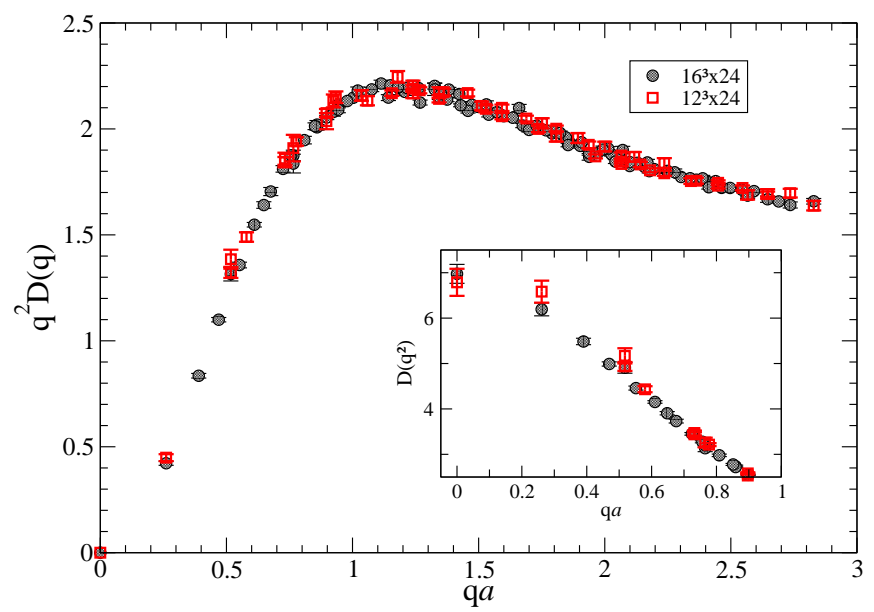

Fig. 5. The gluon dressing function in the vacuum from the $16^{3} \times 24$ and $12^{3} \times 24$ lattice. The inset shows the gluon propagator for infrared momenta. A cylinder cut has been applied to the data to reduce lattice spacing artefacts.

We have fixed our gauge configurations to the minimal Landau gauge by maximising the gauge fixing functional

$$
F[U, g]=\sum_{x, m u} \operatorname{Tr} U_{\mu}^{g}(x)=\sum_{x, \mu} \operatorname{Tr} g(x) U_{\mu}(x) g^{\dagger}(x+\hat{\mu}),
$$

using the standard overrelaxation algorithm. The Landau gauge condition has been imposed with a precision $\left|\partial_{\mu} A_{\mu}\right|<10^{-10}$. We have not investigated the effect of Gribov copies; this will be left to a future study.

First, in figure 5 we show the gluon propagator and dressing function in the vacuum for our two volumes, $12^{3} \times$ 24 and $16^{3} \times 24$. Comparing the data for the two volumes, we see that finite volume effects are modest for these lattices. In order to reduce ultraviolet lattice artefacts, we have applied a weak cylinder cut 70 . The propagator exhibits the usual infrared suppression observed in other lattice studies.

In figure 6] we show the two lowest Matsubara modes for selected spatial momenta as a function of chemical potential from the $N_{\tau}=24$ lattices for different spatial volumes. We find at most a very mild volume dependence, even for zero spatial momentum, with some indication that the magnetic propagator is slightly smaller on the larger volume. Note that because the available (discrete) momentum values depend on the spatial volume, the selected momenta from the $12^{3}$ and $16^{3}$ lattices do not match precisely, and the discrepancy between the propagator values on the two lattices at nonzero spatial momen- 

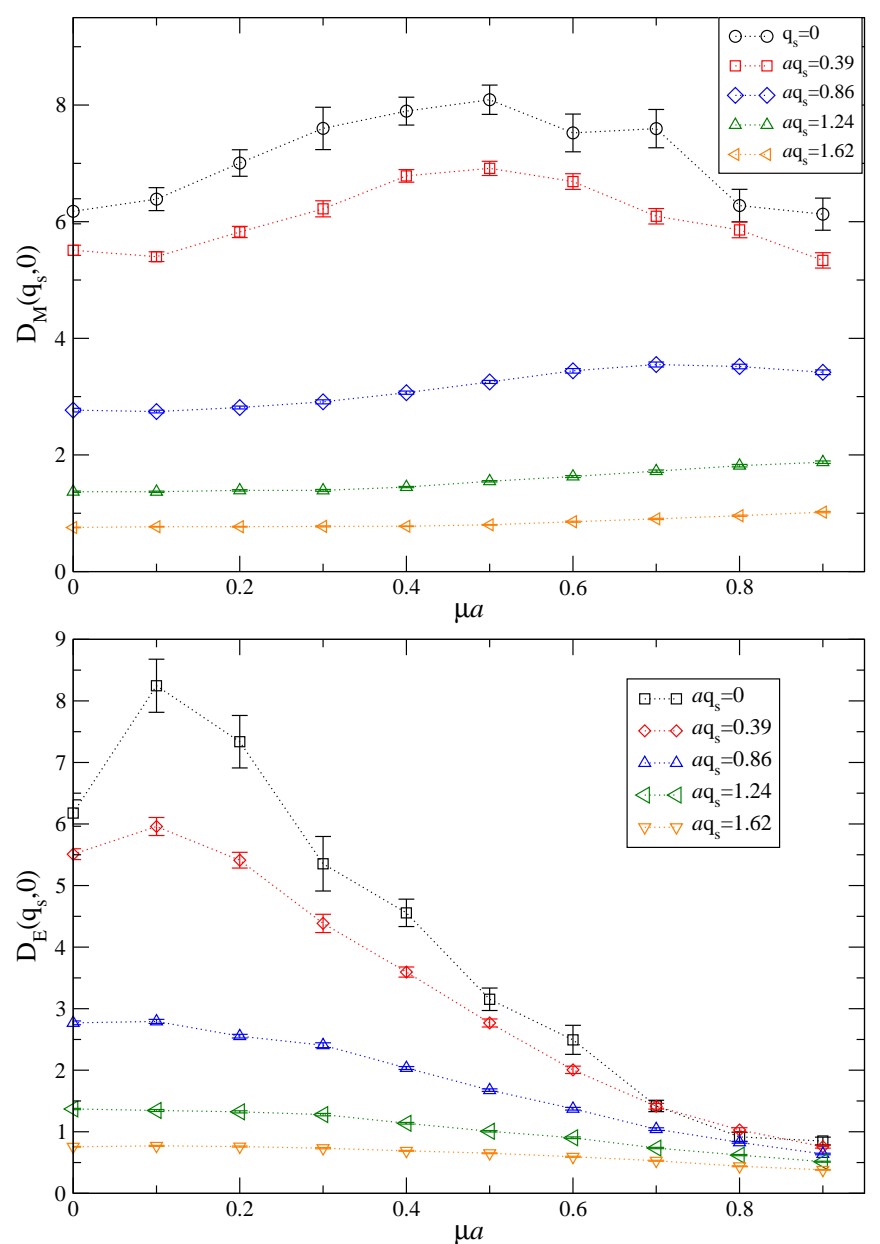

Fig. 7. The zero Matsubara mode of the magnetic (top) and electric (bottom) gluon propagator as a function of chemical potential $\mu$ for selected values of the spatial momentum $q_{s}$, for the $16^{3} \times 8$ lattice.

tum $q_{s}=|\boldsymbol{q}|$ is likely to be at least as much due to the slightly different values of $q_{s}$ as to finite volume effects.

With respect to the infrared suppressed vacuum propagator shown in fig. 5 we find a mild enhancement at intermediate $\mu$, i.e. in the superfluid, confined phase, but a suppression in the deconfined phase, i.e. for large $\mu$ for both tensor structures.

Figure 7 shows the lowest Matsubara mode for the high-temperature, $16^{3} \times 8$ lattice, again as a function of chemical potential and for several different spatial momenta. Here we find a considerably more complex picture. The electric form factor becomes progressively more suppressed with increasing $\mu$ for all momenta, while for the magnetic form factor the lowest momentum modes show an interesting behaviour, with a peak at $\mu a \approx 0.5$. For large spatial momenta this form factor is instead enhanced at large $\mu$.

We now turn to the thermal behaviour of the gluon propagator at fixed chemical potential. Fig. 8 shows the zeroth Matsubara modes of the propagators for $\mu a=0.5$ and $j a=0.04$ on a spatial $16^{3}$ lattice as a function of temperature, with higher modes shown in fig. 9. The magnetic
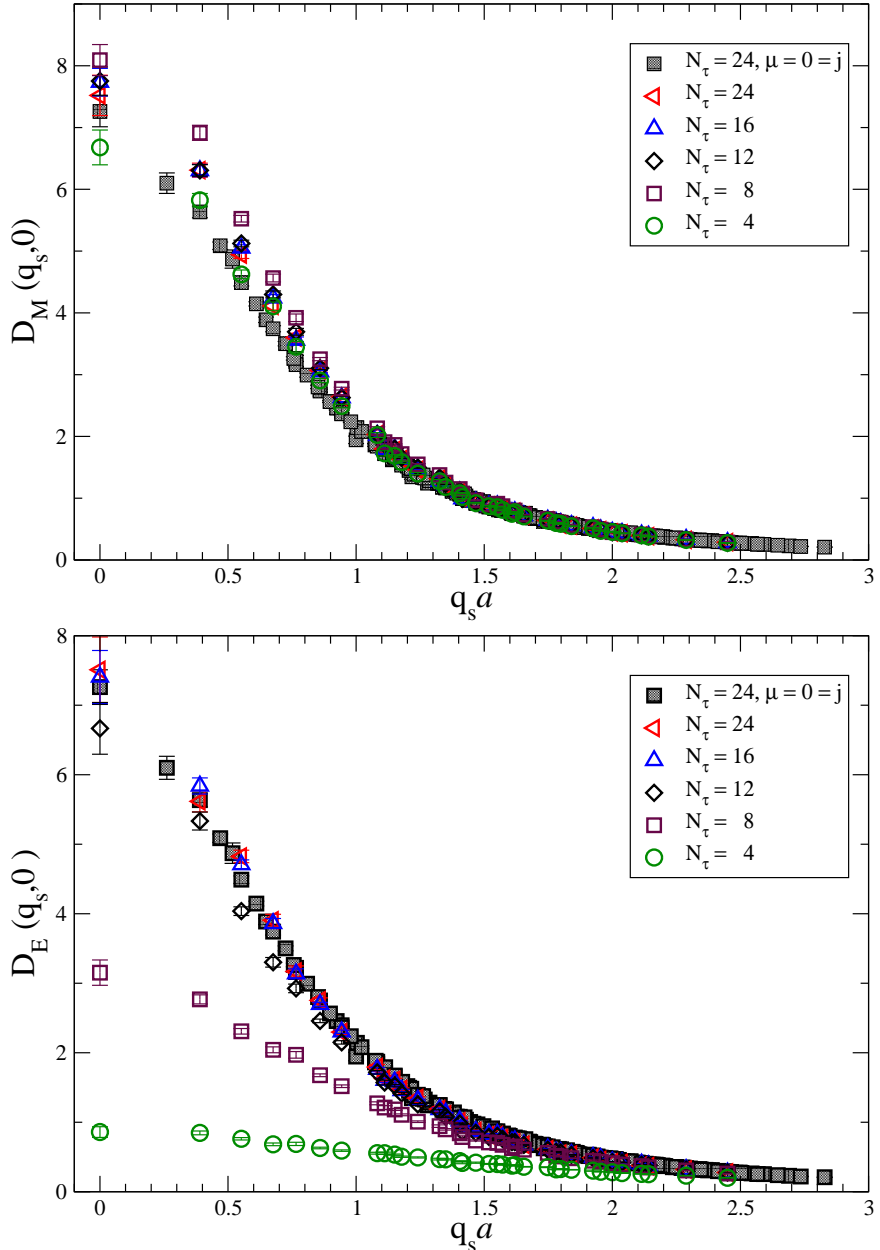

Fig. 8. Thermal behaviour of the zeroth Matsubara mode of the magnetic (top) and electric (bottom) propagators at $\mu a=$ 0.5 and $j a=0.04$ on $16^{3} \times N_{\tau}$ lattices.

component is slightly enhanced by the effect of chemical potential with respect to the vacuum, but interestingly it hardly feels thermal effects over a large range of low to intermediate temperatures. The electric propagator is suppressed for lower temperatures already. In pure gauge theory $[71,72,73,74,75,76,77$ the zero mode of the electric gluon shows strong enhancement for temperatures below the deconfinement transition, whereas the magnetic gluon is screened also below that transition. However fig. 2 entails that the chosen value of $\mu$ drives the system close to the phase transition already at $N_{\tau}=20$, and hence electric gluon enhancement may not be observed here. The interplay of temperature with chemical potential may also trigger the observed non-suppression for the magnetic mode. Our results are however in qualitative agreement with those obtained in a recent study of QCD with twisted-mass fermions 78. A more detailed analysis of both medium effects and in particular their mutual interaction will be presented elsewhere.

To further quantify the variation of the gluon propagator with $T$ and $\mu$ we employ a global fit $D_{M / E}^{\mathrm{fit}}\left(q^{2}\right)$ 

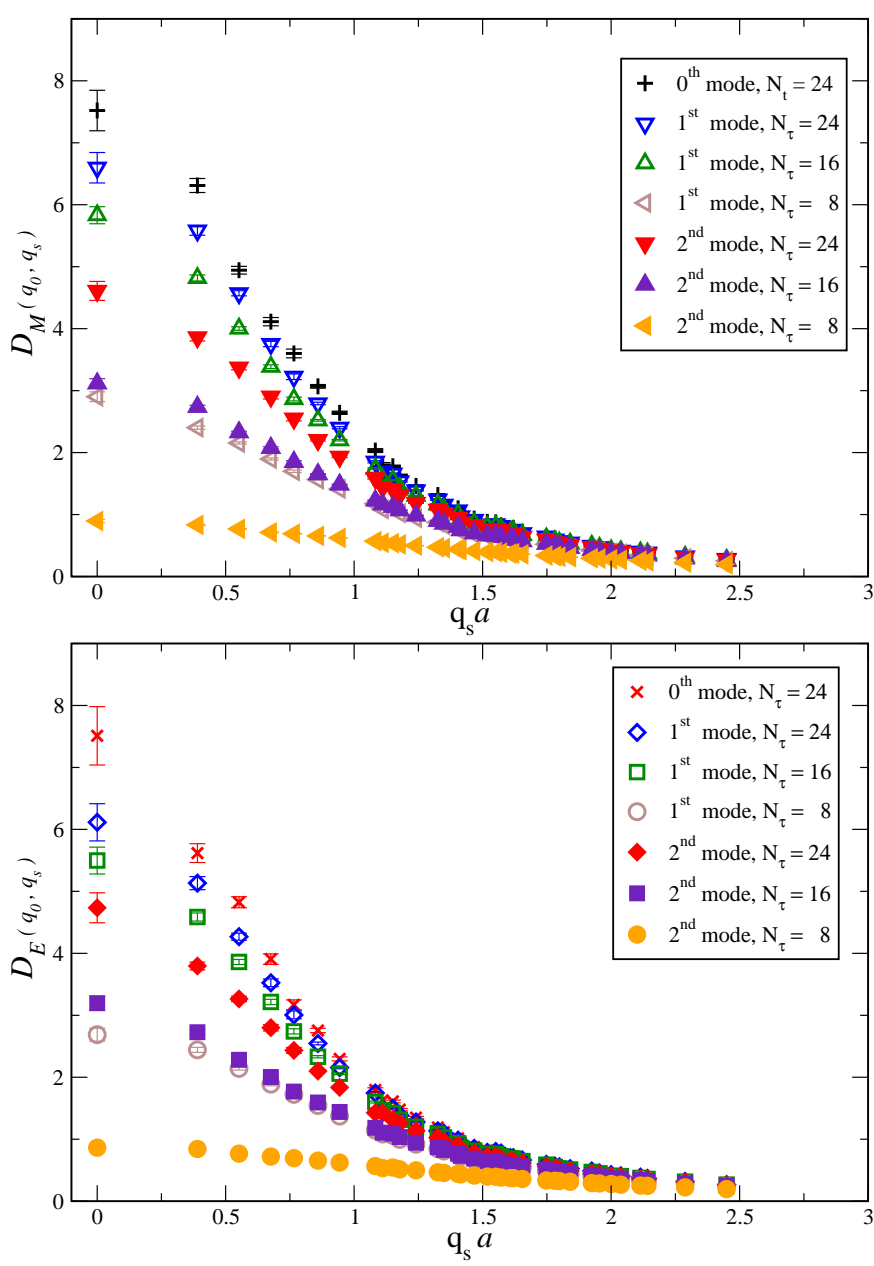

Fig. 9. Thermal behaviour of higher Matsubara modes of the magnetic (top) and electric (bottom) gluon propagators.

to all modes, with $q^{2}=\boldsymbol{q}^{2}+q_{0}^{2}$. The functional form we use is inspired by 72 . However, for the momenta at hand the perturbative running of [72] can be neglected. In the vacuum this gives us a three-parameter fit

$$
D_{M / E}^{\mathrm{fit}}\left(q^{2}\right)=\frac{\Lambda^{2}}{\left(q^{2}+\Lambda^{2}\right)^{2}}\left(q^{2}+\Lambda^{2} a_{M / E}\right)^{-b_{M / E}} .
$$

At $\mu=0=j$ we find $\Lambda a=0.999(3), a_{M}=a_{E}=6.85(3)$ and $b_{M}=b_{E}=-1.031(2)$ on the $16^{3} \times 24$ lattice, with a $\chi^{2}$ per degree of freedom of around 8 for the magnetic mode and 5 for the electric mode. There is a slight volume dependence, with the $12^{3} \times 24$ lattice yielding $\Lambda=0.961(5)$. The normalisation $\Lambda$ is taken to be independent of $T$ and $\mu$, but medium effects modify $a_{M / E}$ and $b_{M / E}$ for magnetic and electric modes individually. The results for the fit parameters are shown in fig. 10 as functions of $N_{\tau}$ on the $16^{3} \times N_{\tau}$ lattices at $\mu a=0.5$ and $j a=0.04$, and in fig. 11 as functions of $\mu$ and lattice volume. For the available data we have found the dependence on $j$ to be weak. At $N_{\tau}=5$ we did not obtain any satisfactory fit for the magnetic form factor, so these points are absent from fig. 10.

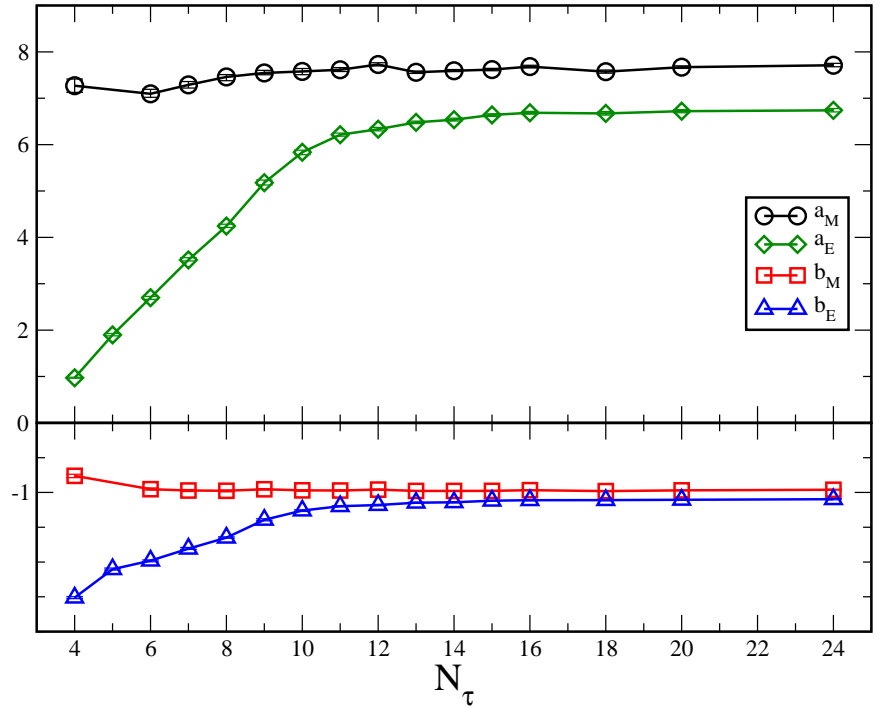

Fig. 10. $N_{\tau}$ dependence of fit parameters $a_{M / E}$ and $b_{M / E}$ for $16^{3} \times N \tau$ lattices at $\mu a=0.5$ and $j a=0.04$.

To illustrate the quality of these fits, fig. 12 shows the fits for $\mu a=0.5, j a=0.04$ on the $16^{3} \times 24$ lattice. We see that (8) gives a reasonable description for both modes, but the underlying Ansatz that the gluon propagator is a function of the four-momentum $q^{2}$ only, works less well for the magnetic form factor. The $\chi^{2} / N_{d f}$ is around 10 for most fits, with the electric form factor giving in general a somewhat better $\chi^{2}$. We are also planning to employ fit models inspired by hard dense loop perturbation theory, which depend separately on $q_{s}$ and $q_{0}$.

\section{Conclusions}

We have carried out a detailed investigation of several aspects of 2-colour, 2-flavour QCD with $m_{\pi} / m_{\rho} \approx 0.8$ at nonzero temperature $T$ and quark chemical potential $\mu$. Our main findings are summarised below.

1. We have located the superfluid to normal and deconfinement transitions in the region $0.35 \leq \mu a \leq 0.6$ $(\mu=385-665 \mathrm{MeV})$. The superfluid to normal transition temperature $T_{s}$ is remarkably constant in this region, while the deconfinement temperature $T_{d}$ shows a decrease with $\mu$ which appears to continuously connect to the $\mu=0$ transition identified in 7]. It also appears to extrapolate smoothly to the high- $\mu$, low- $T$ transition previously observed [5,6,7, although in the absence on any accurate data for the Polyakov loop at low temperature this must be taken merely as indicative.

2. The superfluid to normal transition appears to behave like a second order phase transition, while the deconfinement transition looks like a smooth crossover, which becomes broader with increasing $\mu$. This would have to be backed up with a careful finite volume and critical scaling analysis. 

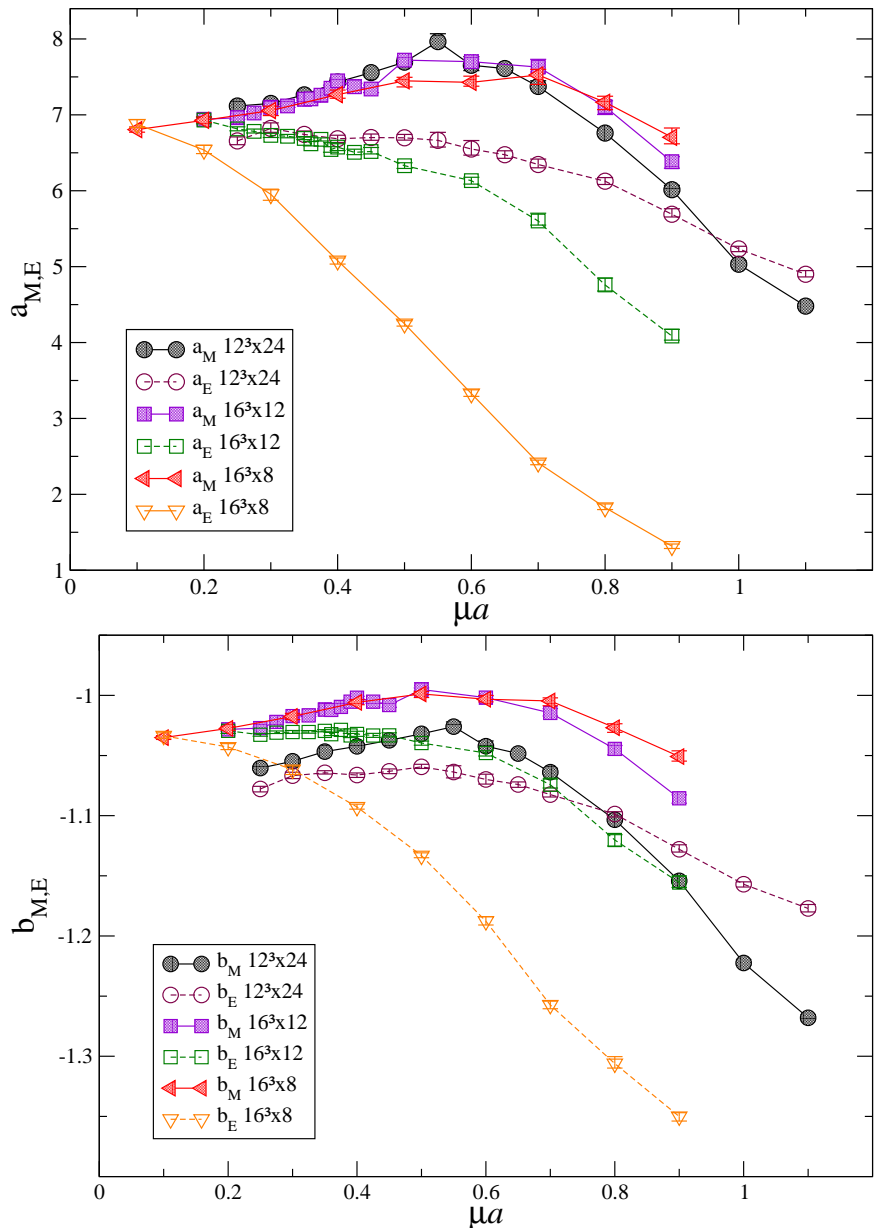

Fig. 11. Dependence of fit parameters $a_{M / E}$ (top) and $b_{M / E}$ (bottom) on $\mu$ and lattice volume.

3. The static quark potential at low temperature is at most only very weakly screened at large $\mu$, suggesting that the dense medium with $\langle L\rangle \neq 0$ is not an ordinary, deconfined quark-gluon plasma.

4. The electric (longitudinal) gluon propagator in Landau gauge becomes strongly screened with increasing temperature and chemical potential. The magnetic (transverse) gluon shows little sensitivity to temperature, and exhibits a mild enhancement at intermediate $\mu$ before coming suppressed at large $\mu$.

The structure of the phase diagram is summarised in fig. 13. We also include the estimates for the deconfinement transition given in 7. We see that these are consistent with our new estimates in this paper. The indications are that the deconfinement line crosses the superfluid to normal transition line, giving rise to a region of deconfined, superfluid matter, but in the absence of precise data at larger $\mu$, we can not say this with any degree of certainty. To map out the phase boundaries with greater precision and to clearly establish the order of the transitions, large-scale simulations on several spatial volumes will be required. This goes beyond our current computa-
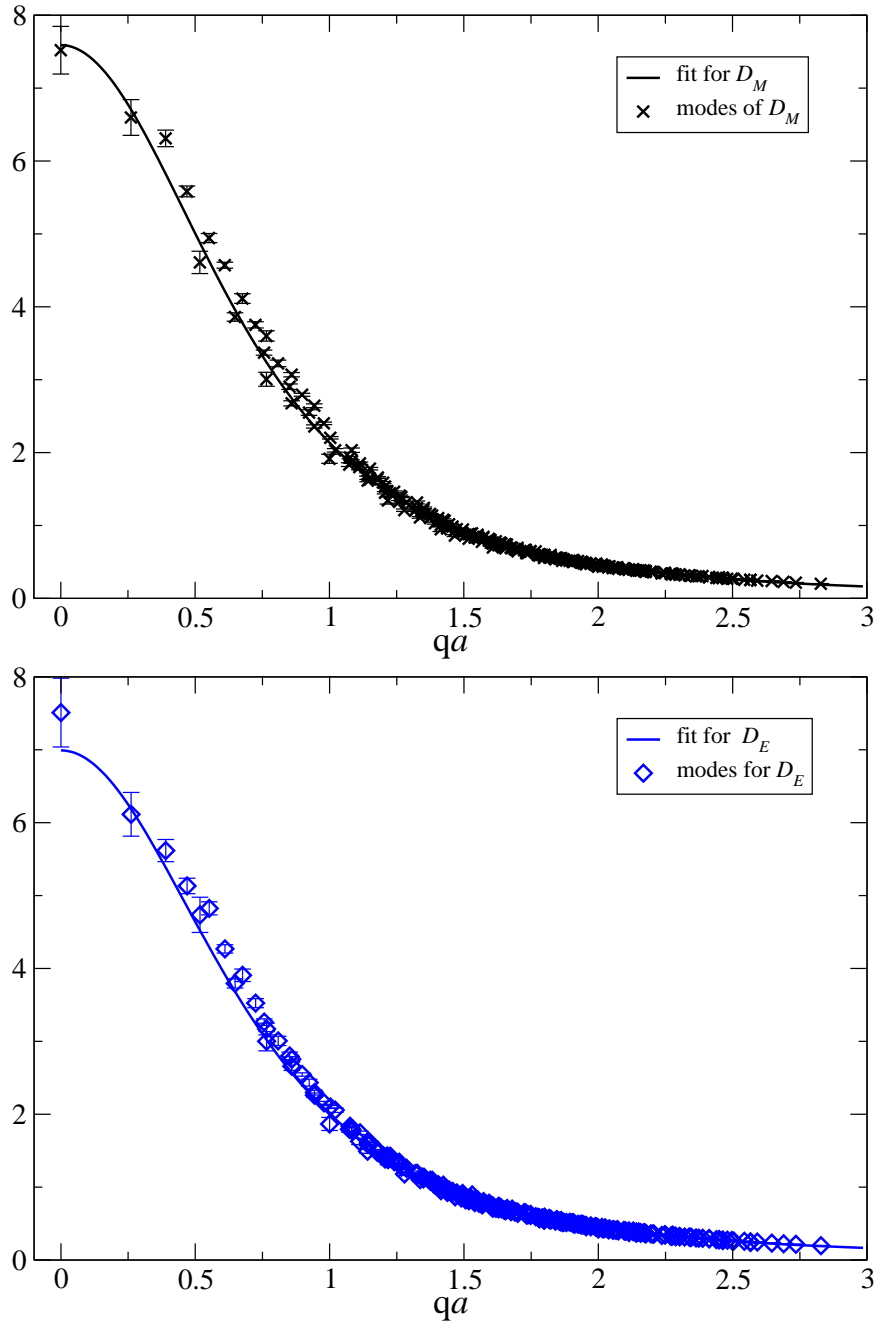

Fig. 12. Multimodal fits of the form eq. (8) for the magnetic (top) and electric (bottom) gluon propagators at $a \mu=0.5$ and $a j=0.04$ on the $16^{3} \times 24$ lattice. Note that the functions are plotted versus four-momentum $q$ on the abscissa.

tional capabilities, but is an interesting topic for future investigations.

We should also note that these simulations have been carried out with a rather large quark mass, and it remains to be seen to what extent these features persist as the quark mass is reduced.

The lack of screening (and possibly even antiscreening) observed in the static quark potential requires further investigation to establish whether this is a signal of an exotic state of matter or a result of poor overlap with relevant states and/or lattice artefacts. We are planning to compute the static quark potential at higher temperatures, using both the Wilson loop and Polyakov loop correlator, to further elucidate this.

The screening of the static magnetic gluon propagator (zero Matsubara mode) at high $\mu$ and low $T$ is a clear signal of the breakdown of resummed perturbation theory, which predicts that this mode is unscreened to all orders. This is likely to be relevant also for real QCD, and should 


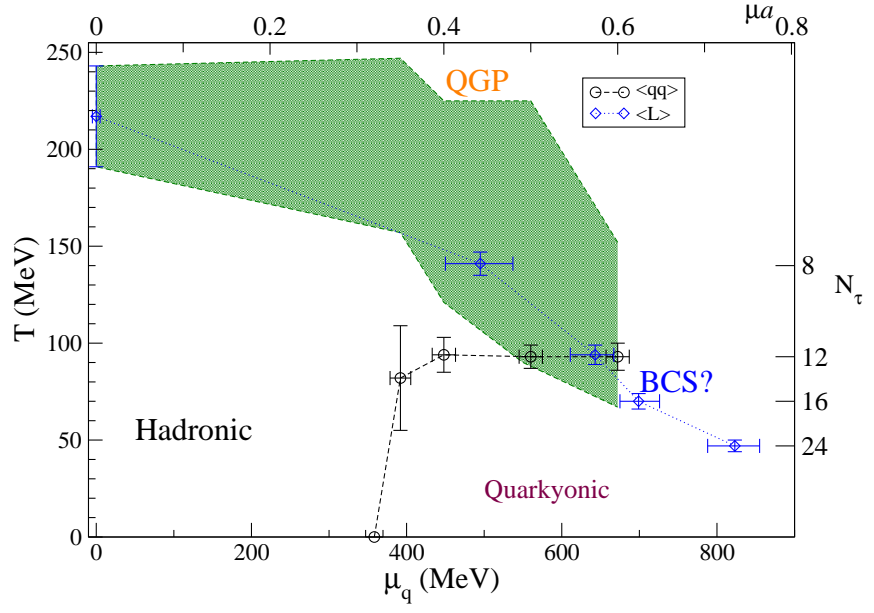

Fig. 13. Phase diagram of $\mathrm{QC}_{2} \mathrm{D}$ with $m_{\pi} / m_{\rho}=0.8$. The black circles denote the superfluid to normal phase transition; the green band the deconfinement crossover. The blue diamonds are the estimates for the deconfinement line from [7.

be taken into account in any future study using model gluon propagators to compute for example the superfluid or superconducting gap.

In an ongoing study we will compare the lattice gluon propagator at non-vanishing chemical potential and temperature with results from functional continuum methods, extending studies of thermal propagators $79,80,81$ to finite density. As there are no insurmountable problems in $\mathrm{QC}_{2} \mathrm{D}$, in particular no sign problem, a direct analysis serves to identify possible technical limitations in either method, stemming from finite size and finite volume artefacts in the lattice formulation, or from inevitably necessary truncations in the continuum description. We are also in the process of computing the quark propagator, which will give further input to these studies.

We are currently extending our study of $\mathrm{QC}_{2} \mathrm{D}$ to smaller lattice spacings, which will enable us to perform a controlled extrapolation to the continuum limit and clarify the possible role of lattice artefacts at large $\mu$.

This work is carried out as part of the UKQCD collaboration and the DiRAC Facility jointly funded by STFC, the Large Facilities Capital Fund of BIS and Swansea University. We thank the DEISA Consortium (www.deisa.eu), funded through the EU FP7 project RI-222919, for support within the DEISA Extreme Computing Initiative. The simulation code was adapted with the help of Edinburgh Parallel Computing Centre funded by a Software Development Grant from EPSRC. We acknowledge the use of the USQCD cluster at Fermilab for part of this work. The work has been carried out with the support of Science Foundation Ireland grant 11-RFP.1-PHY3193. DM is supported by U.S. Department of Energy grant under contract no. DE-FG02-85ER40237. We thank Pietro Giudice, Simon Hands and Jan Pawlowski for stimulating discussions and advice.

\begin{tabular}{ccr|lllc} 
Fit & $\mu a$ & $N_{\tau}$ & $A$ & $B$ & $\alpha$ & $\chi^{2} / N_{d f}$ \\
\hline (9) & 0.4 & 9 & $0.0036(1)$ & $0.695(3)$ & & 26 \\
& 0.4 & 10 & $0.0056(1)$ & $0.711(4)$ & & 102 \\
& 0.4 & 11 & $0.0096(1)$ & $0.646(4)$ & & 9.8 \\
& 0.5 & 9 & $0.0037(2)$ & $0.829(4)$ & & 51 \\
& 0.5 & 10 & $0.0095(2)$ & $0.790(6)$ & & 54 \\
& 0.5 & 11 & $0.0155(2)$ & $0.712(6)$ & & 45 \\
& 0.6 & 9 & $0.0034(2)$ & $0.966(4)$ & & 46 \\
& 0.6 & 10 & $0.0095(2)$ & $0.976(6)$ & & 48 \\
& 0.6 & 11 & $0.0203(2)$ & $0.849(6)$ & & 180 \\
\hline (10) & 0.4 & 9 & & $0.490(8)$ & $0.852(5)$ & 7.4 \\
& 0.4 & 10 & & $0.438(7)$ & $0.793(5)$ & 40 \\
& 0.4 & 11 & & $0.321(5)$ & $0.683(5)$ & 11 \\
& 0.5 & 9 & & $0.617(10)$ & $0.875(5)$ & 28 \\
& 0.5 & 10 & & $0.439(9)$ & $0.734(6)$ & 9.4 \\
& 0.5 & 11 & & $0.315(6)$ & $0.612(6)$ & 1.6 \\
& 0.6 & 9 & & $0.746(11)$ & $0.893(5)$ & 20 \\
& 0.6 & 10 & & $0.576(9)$ & $0.767(5)$ & 19 \\
0.6 & 11 & & $0.357(6)$ & $0.584(5)$ & 55 \\
\hline (11) & 0.4 & 9 & $-0.003(1)$ & $0.76(4)$ & $0.40(3)$ & 8.7 \\
& 0.4 & 10 & $-0.019(4)$ & $0.47(4)$ & $0.24(1)$ & 3.2 \\
& 0.4 & 11 & $0.003(3)$ & $0.85(6)$ & $0.45(4)$ & $*$ \\
& 0.5 & 9 & $-0.008(2)$ & $0.70(4)$ & $0.42(3)$ & 29 \\
0.5 & 10 & $-0.012(4)$ & $0.55(5)$ & $0.31(3)$ & $*$ \\
& 0.5 & 11 & $-0.005(4)$ & $0.54(5)$ & $0.28(2)$ & 0.88 \\
0.6 & 9 & $-0.010(2)$ & $0.69(3)$ & $0.48(3)$ & 0.05 \\
0.6 & 10 & $-0.006(4)$ & $0.68(6)$ & $0.59(6)$ & $*$ \\
& 0.6 & 11 & $-0.055(12)$ & $0.28(4)$ & $0.269(4)$ & $*$ \\
\hline
\end{tabular}

Table 4. Parameters for the $j \rightarrow 0$ extrapolation of $\langle q q\rangle$ in the transition region, using a linear (9), power-law (10) and power + constant (11) Ansatz. All four values of $j$ have been used. Where there is a star in the column for $\chi^{2} / N_{d f}$, the central fit value was outside the $68 \%$ confidence interval, and the quoted value is instead taken to be in the middle of the $68 \%$ confidence interval.

\section{A Diquark source extrapolation}

We have used 3 different functional forms to extrapolate the diquark condensate to zero diquark source,

$$
\begin{array}{ll}
\text { linear: } & \langle q q\rangle=A+B j, \\
\text { power law: } & \langle q q\rangle=B j^{\alpha}, \\
\text { constant + power: } & \langle q q\rangle=A+B j^{\alpha} .
\end{array}
$$

The results of these extrapolations are summarised in table 4. We find that the linear form is clearly disfavoured; however, the pure power law does not give a good fit either in most cases, while the constant + power Ansatz is unstable and tends to give a negative intercept at $j=0$. Clearly, more work is needed to obtain good control over the $j \rightarrow 0$ extrapolation.

\section{References}

1. G. Aarts, F. A. James, E. Seiler and I.-O. Stamatescu, Eur.Phys.J. C71, 1756 (2011) [arXiv:1101.3270].
2. G. Aarts, PoS LATTICE2012, 017 [arXiv:1302.3028]. 
3. S. Chandrasekharan, Phys.Rev. D86, 021701 (2012) [arXiv:1205.0084].

4. AuroraScience Collaboration, M. Cristoforetti, F. Di Renzo and L. Scorzato, Phys.Rev. D86, 074506 (2012) [arXiv:1205.3996].

5. S. Hands, S. Kim and J.-I. Skullerud, Eur. Phys. J. C48, 193 (2006) [hep-lat/0604004].

6. S. Hands, S. Kim and J.-I. Skullerud, Phys. Rev. D81, 091502R (2010) [arXiv:1001.1682].

7. S. Cotter, P. Giudice, S. Hands and J.-I. Skullerud, Phys.Rev. D87, 034507 (2013) [arXiv:1210.4496].

8. A. Maas, L. von Smekal, B. Wellegehausen and A. Wipf, Phys.Rev. D86, 111901 (2012) [arXiv:1203.5653].

9. J. B. Kogut and D. K. Sinclair, Phys. Rev. D66, 034505 (2002) [hep-lat/0202028].

10. S. Hands et al., Eur. Phys. J. C17, 285 (2000) [heplat/0006018].

11. S. Hands, I. Montvay, L. Scorzato and J. Skullerud, Eur. Phys. J. C22, 451 (2001) [hep-lat/0109029].

12. P. Giudice and A. Papa, Phys.Rev. D69, 094509 (2004) [arXiv:hep-lat/0401024].

13. P. Cea, L. Cosmai, M. D'Elia and A. Papa, JHEP 0702, 066 (2007) [arXiv:hep-lat/0612018].

14. P. Cea, L. Cosmai, M. D'Elia, C. Manneschi and A. Papa, Phys.Rev. D80, 034501 (2009) [arXiv:0905.1292].

15. J. Kogut, M. Stephanov and D. Toublan, Phys. Lett. B464, 183 (1999) [hep-ph/9906346].

16. J. Kogut, M. Stephanov, D. Toublan, J. Verbaarschot and A. Zhitnitsky, Nucl. Phys. B582, 477 (2000) [hep$\mathrm{ph} / 0001171]$.

17. K. Splittorff, D. Son and M. Stephanov, Phys. Rev. D64, 016003 (2001) [hep-ph/0012274].

18. J. Lenaghan, F. Sannino and K. Splittorff, Phys.Rev. D65, 054002 (2002) [arXiv:hep-ph/0107099].

19. K. Splittorff, D. Toublan and J. Verbaarschot, Nucl.Phys. B620, 290 (2002) [arXiv:hep-ph/0108040].

20. J. B. Kogut, D. Toublan and D. Sinclair, Phys. Lett. B514, 77 (2001) [hep-lat/0104010].

21. J. B. Kogut, D. Toublan and D. K. Sinclair, Nucl. Phys. B642, 181 (2002) [hep-lat/0205019].

22. K. Splittorff, D. Toublan and J. Verbaarschot, Nucl.Phys. B639, 524 (2002) [arXiv:hep-ph/0204076].

23. G. V. Dunne and S. M. Nishigaki, Nucl.Phys. B654, 445 (2003) [arXiv:hep-ph/0210219]

24. G. V. Dunne and S. M. Nishigaki, Nucl.Phys. B670, 307 (2003) [arXiv:hep-ph/0306220].

25. T. Brauner, Mod.Phys.Lett. A21, 559 (2006) [arXiv:hep$\mathrm{ph} / 0601010]$.

26. T. Kanazawa, T. Wettig and N. Yamamoto, JHEP 0908, 003 (2009) [arXiv:0906.3579].

27. T. Kanazawa, T. Wettig and N. Yamamoto, Phys.Rev. D81, 081701 (2010) [arXiv:0912.4999].

28. T. Kanazawa, T. Wettig and N. Yamamoto, JHEP 1112 , 007 (2011) [arXiv:1110.5858].

29. M. Giannini, L. Kondratyuk and M. Krivoruchenko, (1991).

30. L. Kondratyuk and M. Krivoruchenko, Z.Phys. A344, 99 (1992).

31. R. Rapp, T. Schäfer, E. V. Shuryak and M. Velkovsky, Phys.Rev.Lett. 81, 53 (1998) [arXiv:hep-ph/9711396].

32. C. Ratti and W. Weise, Phys. Rev. D70, 054013 (2004) [arXiv:hep-ph/0406159].
33. G.-F. Sun, L. He and P. Zhuang, Phys.Rev. D75, 096004 (2007) [arXiv:hep-ph/0703159].

34. T. Brauner, K. Fukushima and Y. Hidaka, Phys. Rev. D80, 074035 (2009) [arXiv:0907.4905].

35. J. O. Andersen and T. Brauner, Phys. Rev. D81, 096004 (2010) [arXiv:1001.5168].

36. M. Harada, C. Nonaka and T. Yamaoka, Phys.Rev. D81, 096003 (2010) [arXiv:1002.4705].

37. T. Zhang, T. Brauner and D. H. Rischke, JHEP 1006, 064 (2010) [arXiv:1005.2928]

38. L. He, Phys.Rev. D82, 096003 (2010) [arXiv:1007.1920].

39. N. Strodthoff, B.-J. Schaefer and L. von Smekal, Phys.Rev. D85, 074007 (2012) [arXiv:1112.5401].

40. J. C. R. Bloch, C. D. Roberts and S. M. Schmidt, Phys. Rev. C60, 065208 (1999) [arXiv:nucl-th/9907086].

41. A. Nakamura, Phys.Lett. B149, 391 (1984).

42. S. Hands, J. B. Kogut, M.-P. Lombardo and S. E. Morrison, Nucl. Phys. B558, 327 (1999) [hep-lat/9902034].

43. J. B. Kogut, D. K. Sinclair, S. J. Hands and S. E. Morrison, Phys. Rev. D64, 094505 (2001) [hep-lat/0105026].

44. S. Muroya, A. Nakamura and C. Nonaka, Nucl. Phys. Proc. Suppl. 119, 544 (2003) [hep-lat/0208006].

45. S. Chandrasekharan and F.-J. Jiang, Phys.Rev. D74, 014506 (2006) [arXiv:hep-lat/0602031].

46. B. Allés, M. D'Elia and M. Lombardo, Nucl.Phys. B752, 124 (2006) [arXiv:hep-lat/0602022].

47. M.-P. Lombardo, M. L. Paciello, S. Petrarca and B. Taglienti, Eur.Phys.J. C58, 69 (2008) [arXiv:0804.4863].

48. S. Hands, P. Kenny, S. Kim and J.-I. Skullerud, Eur.Phys.J. A47, 60 (2011) [arXiv:1101.4961].

49. T. D. . Cohen, Phys.Rev.Lett. 91, 222001 (2003) [arXiv:hep-ph/0307089].

50. L. McLerran and R. D. Pisarski, Nucl. Phys. A796, 83 (2007) [arXiv:0706.2191].

51. T. Kojo, Y. Hidaka, L. McLerran and R. D. Pisarski, Nucl. Phys. A843, 37 (2010) [arXiv:0912.3800].

52. J. Braun, H. Gies and J. M. Pawlowski, Phys.Lett. B684, 262 (2010) [arXiv:0708.2413].

53. F. Marhauser and J. M. Pawlowski, arXiv:0812.1144.

54. J. Braun, A. Eichhorn, H. Gies and J. M. Pawlowski, Eur.Phys.J. C70, 689 (2010) [arXiv:1007.2619].

55. L. Fister and J. M. Pawlowski, arXiv:1301.4163.

56. J.-I. Skullerud, Nucl. Phys. A820, 175c (2009) [arXiv:0810.3795].

57. J.-I. Skullerud, PoS QCD-TNT09, 043 [arXiv:0912.0844].

58. S. Cotter et al., [arXiv:1210.6757].

59. K. G. Wilson, Phys.Rev. D10, 2445 (1974).

60. M. Laine, O. Philipsen, P. Romatschke and M. Tassler, JHEP 0703, 054 (2007) [arXiv:hep-ph/0611300].

61. N. Brambilla, J. Ghiglieri, A. Vairo and P. Petreczky, Phys.Rev. D78, 014017 (2008) [arXiv:0804.0993].

62. A. Beraudo, J.-P. Blaizot and C. Ratti, Nucl.Phys. A806, 312 (2008) [arXiv:0712.4394].

63. A. Beraudo, J. Blaizot, P. Faccioli and G. Garberoglio, Nucl.Phys. A846, 104 (2010) [arXiv:1005.1245].

64. A. Bazavov and P. Petreczky, Nucl.Phys.A904-905 2013, 599c (2013) [arXiv:1210.6314].

65. T. Kawanai and S. Sasaki, Phys.Rev. D85, 091503 (2012) [arXiv:1110.0888].

66. C. Allton, W. Evans and J.-I. Skullerud, PoS LATTICE2012, 082 (2012) [arXiv:1306.3140]; arXiv:1303.5331. 
67. S. Hands, S. Kim and J.-I. Skullerud, Phys.Lett. B711, 199 (2012) [arXiv:1202.4353].

68. A. Bazavov and P. Petreczky, arXiv:1303.5500.

69. SESAM Collaboration, G. S. Bali, H. Neff, T. Düssel, T. Lippert and K. Schilling, Phys.Rev. D71, 114513 (2005) [arXiv:hep-lat/0505012].

70. UKQCD, D. B. Leinweber, J. I. Skullerud, A. G. Williams and C. Parrinello, Phys. Rev. D60, 094507 (1999) [heplat/9811027].

71. A. Cucchieri, A. Maas and T. Mendes, Phys.Rev. D75, 076003 (2007) [arXiv:hep-lat/0702022].

72. C. S. Fischer, A. Maas and J. A. Muller, Eur. Phys. J. C68, 165 (2010) [arXiv:1003.1960]

73. V. Bornyakov and V. Mitrjushkin, Int.J.Mod.Phys. A27, 1250050 (2012) [arXiv:1103.0442].

74. A. Maas, Phys. Rep. in press (2013) [1106.3942].

75. R. Aouane et al., Phys.Rev. D85, 034501 (2012) [arXiv:1108.1735].

76. A. Maas, J. M. Pawlowski, L. von Smekal and D. Spielmann, Phys.Rev. D85, 034037 (2012) [arXiv:1110.6340].

77. A. Cucchieri and T. Mendes, PoS FACESQCD, 007 (2010) [arXiv:1105.0176].

78. R. Aouane, F. Burger, E.-M. Ilgenfritz, M. MullerPreussker and A. Sternbeck, Phys. Rev. D 87, 114502 (2013) [arXiv:1212.1102].

79. L. Fister and J. M. Pawlowski, arXiv:1112.5440.

80. L. Fister and J. M. Pawlowski, arXiv:1112.5429.

81. L. Fister, PhD thesis, Heidelberg University (2012). 